\title{
Advanced technologies for sustainable building in the protected areas: two case studies in Italy
}

\author{
F. Cinquepalmi, F. Cumo, F. Gugliermetti \& V. Sforzini \\ Department of Fisica Tecnica, Sapienza University of Rome, Italy
}

\begin{abstract}
For some years now the General Directorate for the Protection of Nature of the Italian Ministry for the Environment, Land and Sea, and the Department of "Fisica Tecnica" of the "Sapienza" University of Rome have been collaborating on issues such as sustainable development in protected areas and new technologies concerning innovative low-impact materials to be used in areas of particularly high environmental sensitivity.

The first case study presented for the Protected Areas deals with a pilot project in a suburban green area jointly individuated with the "Assessorato all'Ambiente" of the Municipality of Rome designed to evaluate low-impact building in contexts of environmental "Excellency", such as parkland in urban areas, city outskirts, green areas and national parks. In line with the Strategic Environmental Evaluation, the reversibility of such buildings has been evaluated with particular care, although other considerations, such as aspects related to the education towards sustainability in architecture, were also looked into.

Research has been oriented towards pinpointing 'new' envelope technologies in which to assembly components and technical solutions already known as "sustainable" and/or "energy efficient" (such as coat insulation, roof garden, ecologically compatible materials such as wood, etc.), but whose combined application has not yet been tested as a whole complex system.

During the realization of this $500 \mathrm{mq}$ eco-sustainable structures the following technologies have been included: wooden structures and sheathing for a low energy consumption and naturally recyclable material; green roof coverings; systems for water cycle management; solar collectors for hot water production and photovoltaic systems; high performance glass in terms of light transmission, solar control and thermal insulation.

The second case study, coming from the experience of the pilot project, is the realization of a $15 \mathrm{mq}$ infopoint that is energetically self sufficient in the
\end{abstract}


protected area of the Asinara island (Ardito S., Parks of Italy - the protected areas system, Italian Environmental Ministry - Carsa Edizioni, 2004, 226-230 abstract).

This structure is made of a light architecture supporting a controlled and didactical use and fruition of a critical environment; this bungalow is made of a wooden modular shape with low technological equipments, based on the principals of prefabrication and low energy consumption, fed only by $1,5 \mathrm{kWp}$ photovoltaic system integrated into the roof.

In this paper are summarized the first preliminary results of the monitoring campaign actually performed in both structures.

Keywords: sustainable, integrated, green energy, solar energy, energy efficiency, sustainable technologies, natural elements, climatic resource, saving CO2.

\section{Technology for sustainable buildings}

According to a general protocol regarding the Best Available Technique for building construction in the protected areas pointed out by the Department of Fisica Tecnica and the for the Italian Environmental Ministry, the application of the following technologies has been evaluated for the two pilot projects [2].

\subsection{Wooden structures and sheathing for a low energy consumption and naturally recyclable material}

Much used in building, wood is prized on account of its resistance and because its structural performance has qualities that are akin to steel. As a low energy material, its production cycle optimises the use of a material that is poor in itself but which offers elements that are not otherwise used because of their size (dimension and transport).

As well as being aesthetically pleasing, wood for structure is also innovative and reliable: Reliable because its entire production process is ruled and continuously monitored. The end result is a product with definite and certifiable performance. Innovative because planning, working, assemblage and joining techniques are in continuous evolution and offer increasingly broad prospects in terms of feasibility and cost containment.

The overall pleasing effect of timber in architecture derives from the fact that the material is selected also with this in mind and is presented in all its naturalness, compact and without defects. Among the many advantages of timber are its excellent thermal, electric and acoustic insulating properties; it is a hygroscopic material that is therefore able to reduce humidity fluctuations from the surrounding environment; an organic material made up of roughly $50 \%$ carbon, $42 \%$ oxygen, $6 \%$ hydrogen, $1 \%$ nitrogen and $1 \%$ other elements.

Timber in buildings has a substantially higher resistance to fire than most other traditional construction materials such as brick or steel. When exposed to fire, the supporting wood elements guarantee a good level of static security for a building and offer a visual warning of fire, not considering chemical fireproofing treatments that can be toxic in the event of combustion. 


\subsection{The application of green roof coverings}

The chronic lack of green in built up areas is an issue with far greater implications than the enjoyment of the inhabitants of a town. The overwhelming presence of asphalt and cement in the reflecting surfaces of buildings in cities concerns both the health of the buildings themselves and the quality of life of town dwellers. Although little can be done to mitigate the over-heating of road surfaces, with the exception of areas used for parking, it is nonetheless both useful and possible work on the asphalt and cement rooftops of buildings.

On a summer day the horizontal surfaces of buildings exposed to the sun can reach temperatures of up to $80^{\circ} \mathrm{C}$, with temperatures dropping to as low as $20^{\circ} \mathrm{C}$ at night. This means that there is a temperature difference of around $60^{\circ} \mathrm{C}$. In wintertime the temperature of building surfaces in northern Italy can drop to around $-20^{\circ} \mathrm{C}$, which means that in a year the surface temperature of a building can vary by $100^{\circ} \mathrm{C}$ or more.

Besides the structural stress such temperature variations subject buildings to, the phenomenon of general heating in urban areas entails rising costs through the use of conditioners and consequently further overheating from the emissions of convectors. This generates the phenomenon known as "urban heat islands", which the Department "Fisica Tecnica" has been looking into also with studies aimed at pinpointing ways in which it can be mitigated. The green covering of flat-roofed buildings provides a number of key services for urban contexts. As well as contributing to the production of oxygen in built up areas, such coverings act as filters for fine dust particulate produced by traffic and reduce noise pollution. They also act as a natural attenuator for the variations in temperature described above: up to $25^{\circ} \mathrm{C}$ between night and day in the summer and a minimum of $-10^{\circ} \mathrm{C}$ during winter. One must also consider the recreational value such coverings offer to citizens, both in terms of direct and indirect fruition, through access to pleasant and ventilated green areas in areas where the presence of high rise buildings necessarily means that green areas at street level would not enjoy the same advantages.

\subsection{Overall water cycle}

Despite being the third country in the world in terms of water consumption after Canada and the United States, for four months a year (June to September) in Italy roughly $15 \%$ of the population - which corresponds to eight million people - receives less than the accepted minimum daily water supply of 50 litres a day per person. Italy's water resources are absorbed $60 \%$ by the country's agricultural sector, followed by $25 \%$ for energy and industry and $15 \%$ for civilian use. The most indicative figure, however, is that the country's entire water needs are covered by the drinkable mains water system, which entails vast costs both in social and financial terms. Most of the consumption is taken up by non-drinkable uses such as WC flushing, watering and household appliances such as dishwashers and washing machines. Up to $50 \%$ of water consumption in this sector could be replaced by stored rainwater or recycled water. 
Systems for storing rainwater provide water for a number of uses and permit considerable saving of drinkable water in private homes, public buildings and in industry. Trapping rainwater also reduces the risk of damage from heavy rain, which otherwise ends up in water purifying through the sewers, considerably reducing their purifying capacity. Rainwater trapping systems are essentially made up of three parts: cistern, a filter and a pump. Rainwater runs off the roof and is channelled by a system of drainpipes into a collecting tank. From here the water runs through a filter into cisterns with a float that stops more water pouring in when the full mark has been reached and that are protected against small animals entering.

A submerged pump carries the water into a decanting tank, whereupon the water can be used for the household's nondrinking needs. Spacer permitting, human sewage can also be channelled into constructed wetland systems, which are relatively easy to install in tanks placed in holes dug into the soil and equipped with filter grills that can be inspected. These are then filled with gravel and planted with plant species with known filtering properties. These simple systems provide water that can be used for watering or reintroduced into the water cycle of the sustainable household as a means for the water closet.

A sustainable integrated water cycle clearly implies separate plumbing systems for drinkable and non-drinkable water, which entails adaptation costs for existing buildings. Such costs can be considerably reduced if separate plumbing systems are incorporated into the initial design of new buildings.

\subsection{Solar collectors for the hot water production and photovoltaic systems}

Within the framework of a generalised reduction in energy consumption, a number of local administrations in Italy have approved incentives for the energy sector in civilian urban developments. The City of Rome in particular has deliberated that all new constructions must be provided with renewable energy sources. All newly constructed buildings for private use must be reliant on renewable energy for $30 \%$ of total consumption and at least $50 \%$ for the production of hot water. The deliberation pays particular attention to the aesthetic aspects of solar panels. Each installation must be evaluated with a view to striking a balance between the best possible exposure to the sun and the need to preserve Rome's historic and artistic heritage.

Thermal panels heat water for domestic use without consuming either gas or electricity.

A first comparison marker of the different technologies available can be obtained by measuring the average quantity of carbon dioxide released into the atmosphere, in comparable conditions, for the production of hot water for domestic use. The energy analysis revealed that the power consumption of a family of four for the production of hot water, using an electric boiler, amounts to $7.74 \mathrm{KWh}$ (electric)/per day. In order to produce one KWh of power, stations in Italy release an average of $0.58 \mathrm{~kg}$ of carbon dioxide $(\mathrm{CO} 2)$, one of the chief gases responsible for the greenhouse effect4. This means that only for its household hot water needs an average family that uses an electrically powered boiler releases roughly $4.5 \mathrm{~kg}$ of $\mathrm{CO} 2 \mathrm{a}$ day into the atmosphere $(1.125 \mathrm{~kg}$ per 
person a day). Methane powered boilers release $0.25 \mathrm{~kg}$ of $\mathrm{CO} 2$ for every thermal kwh produced, which means that a family of four produces $1.74 \mathrm{~kg}$ of $\mathrm{CO} 2$ per day $(0.435 \mathrm{~kg}$ of $\mathrm{CO} 2$ per person a day).

Solar panels providing an integrated source of energy to combine with gas powered boilers offer an interesting alternative today. These combined solar/gas systems ensure the same level of comfort throughout the year, and on latitudes such as Rome's offer a saving of roughly $60 \%$ on the annual consumption of gas. This means that the same family of four can reduce its daily $\mathrm{CO} 2$ emissions to $0.69 \mathrm{~kg}$, for a daily average of $0.174 \mathrm{~kg} / \mathrm{CO} 2$ per person. The production of hot water for domestic use from an electric current passed through the resistance of a boiler is costly in energetic, to gas powered boilers. Where possible, substituting electric boilers with an integrated solar/gas system could therefore be of interest to a high number of domestic and a small number of public consumers.

Photovoltaic systems reduce the energy demand from traditional sources and contribute to the reduction of atmospheric pollution (carbon dioxide emissions that would otherwise be produced by power stations).

Power produced with photovoltaic costs nothing in terms of fuel: for every $\mathrm{kWh}$ produced with this system there is a saving of 250 grams on oil consumption and a reduction of 700 grams of $\mathrm{CO} 2$ emissions, not to mention other gases that contribute to the greenhouse effect. This necessarily translates into an overall environmental and financial advantage. Given that a system of this sort lasts about 30 years and that a small $1.5 \mathrm{kWp}$ system can cover two thirds of the electricity needs of an average Italian family $(2,400 \mathrm{kwh})$, during its lifespan one can estimate that it will produce $60,000 \mathrm{kWh}$. This would save roughly 14 tonnes of fossil fuels and avoid the emission of roughly 40 tonnes of $\mathrm{CO} 2$. In private dwellings and condominiums small solar panels can power appliances that are positioned in areas not served by electricity, such as gardens and the lighting of public spaces.

\subsection{High performance glass in terms of light transmission, solar control and thermal insulation}

A number of technologies including sun radiation control, aeration, passive heat absorption, anti-dazzling, privacy safeguarding, the increase of natural light and so on have been studied for glass in an effort to control and modify the quantity or quality of light. The aim of these new technologies is to optimise and make the most of natural light in indoor spaces by carefully controlling and optimising sunlight.

Special kinds of glass (coloured, reflecting, low-emission, antisolar) can be obtained by using sophisticated technologies and the spreading of oxides or metals over the surface. Together with evolved technology this has enabled an ever greater solar control, thermal insulation and a reduction in energy costs and consumption.

High performance glass is able to control solar radiation, maximising the ratio between light transmission and heat conductibility. Low emission glass keep heat indoors during winter thanks to a very low transmission coefficient and ensures cool during summer thanks to its low solar factor and low energy transmission. 
The study of the performance of transparent building shells is therefore considered essential for containing energy consumption and improving energy efficiency in building.

\section{The pilot project for the municipality of Rome}

This pilot project, located in a $7000 \mathrm{mq}$ area of a Polyvalent Green Point of the Rome Municipality, works with all the described different passive and active technologies, located in the same area, in order to permit the realization of a didactical path where the end users and the scholars could appreciate the renewable energy production and $\mathrm{CO} 2$ saved in real time through electronic screens.

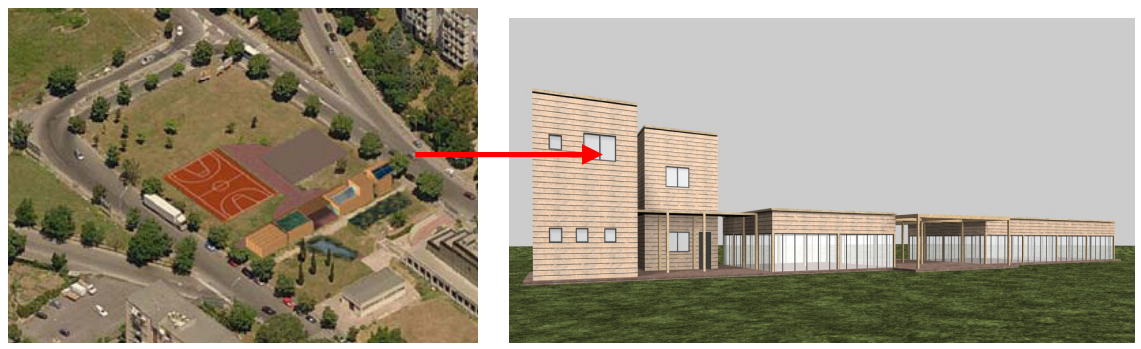

Figure 1: $\quad$ Masterplan and render of Spinaceto building.

The 'active' elements are as follows.

- Solar thermal panels

This system is of a natural circulation kind, with the boiler visible. The panels are flat and the surface corresponds to a minimum module of $2 \mathrm{~m} 2$ of exposed surface and a boiler of roughly 300 litres;

\section{- Solar photovoltaic panels}

The available surface for setting up a photovoltaic system is $12 \mathrm{~m} 2$. Considering the installation of polycrystalline modules and a production of roughly $1 \mathrm{~kW}$ per $8 \mathrm{~m} 2$ of panel the production of electric power will be roughly $1.5 \mathrm{~kW}$, which is enough to cover the energy requirements of communal areas.

\section{- Water cycle (active part)}

Conceived for providing collected rainwater for sanitary use, this system is composed of an underground tank that collects the water that is then pumped into the sanitation system (WC flushing); this system is integrated with the traditional plumbing system that covers the remaining water needs. 


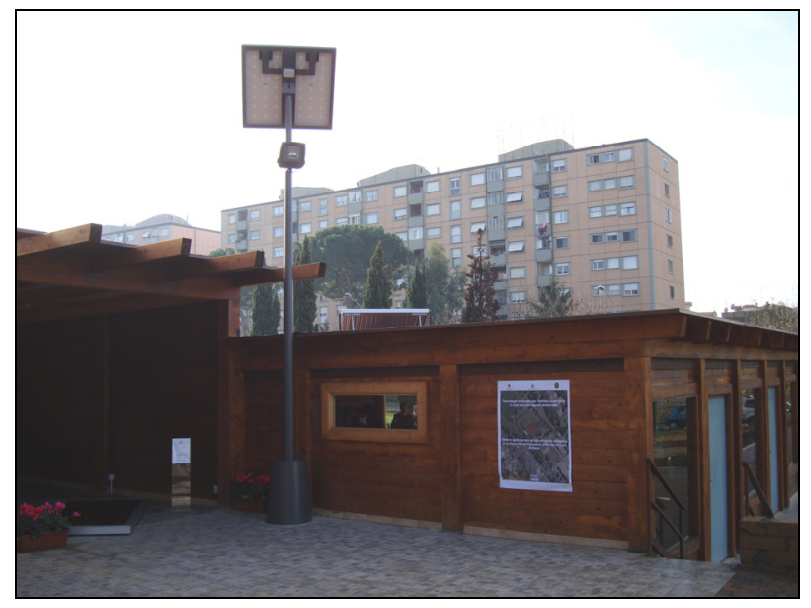

Figure 2: $\quad$ Photovoltaic lighting system.

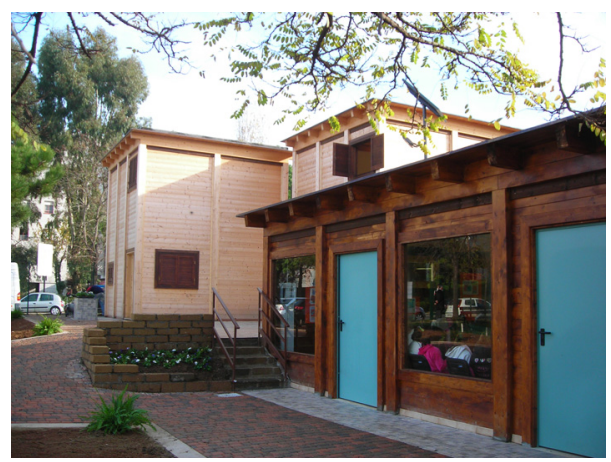

Figure 3: Details of wood vertical closure structures.

The 'passive' elements are as follows.

- Elevation and vertical closure structures

These are made of external cladding: larch staves; air gap; conduction layer: wooden panel; thermal insulation; vapour barrier; vertical structure: laminated panels; internal cladding: wooden panel (spruce pine) [3].

The selected technology consists of a natural material (wooden panels) able to guarantee good seismic resistance coupled with thermal-hygrometric performances according to the D. Lgs. 192/05 and 311/06 (high energy performances in buildings).

\section{- Upper closure with roof garden}

These are made of the following layers: external cladding: grass, bushes and plants; cultivation soil; no woven polyester filtering separation layer; drainage; aeration layer; bituminous primer; insulation layer; vapour barrier; sloping layer; wooden structure. 


\subsection{The foundations}

Wooden structures usually need continuous concrete slab foundation. Because of the structural building system, made with wooden continuous panels, plinths cannot be used. We need to find a continuous foundation system using less concrete than in a regular slab foundation and that requires a minimum excavation of the ground. In order to reduce the landscape impact, the foundations are designed as a concrete riddle along the perimeter of the building. This riddle is not deeply set in the ground thanks to the breadth of the one-storey wooden structure, preserving as much as possible the natural ground surface, thereby reducing the excavation and limiting the use of artificial materials such as concrete. This kind of solution permit an easy removal of the building envelope avoiding substantial changes at the natural landscape so that the same solution can be even applied in high natural value areas such as environmental protected ones.

\subsubsection{The functional elements of the water cycle}

A subsurface flow constructed wetland system has been devised for the building of wastewater treatments; it is composed of two subsequent and contiguous basins localised in the south side of the building. This kind of solution permits to avoid connection with the sewage system, without a long transversal excavation. In fact, the purifying effect is based on microbiological, physical, chemical and aquatic plant processes in the plant - gravel system. The first basin (Subsurfaceflow), roughly $70 \mathrm{~cm}$ deep, should be waterproofed with a layer of clay and filled with different sized gravel without an open water surface. The wastewaters flow under the gravel surface, in order to avoid foetid aerosol. Macrophyte species will be planted in the gravel, with filtering and nutrients interceptive functions. The second basin (surface-flow), has a more educational function, and presents a central open stretch of water surrounded by a border of aquatic plants. Small inspection wells as well as grillage systems permit the monitoring of the water quality and remove non-degradable litter.

\section{The project of the Asinara Island}

The natural park of Asinara Island is one of the most important and interesting environmental protected areas of Italy which hosts some historical buildings used in the past years as hospitals and prisons; consequently the access to the island in the past 200 years has been strongly restricted and the absence of a wide human presence has preserved the biodiversity of this unique Mediterranean location. The difficulties of the described project were in the respect of the strong environmental and building requirements in both the construction and the management phase for the realization of a tourist restoration desk and infopoint in the location of Stretti.

The main goal of the design was the realization of a structure totally removable (no foundation or soil contamination is admitted in the conservation guidelines of the Park) made up of totally natural materials (such as wood) and 


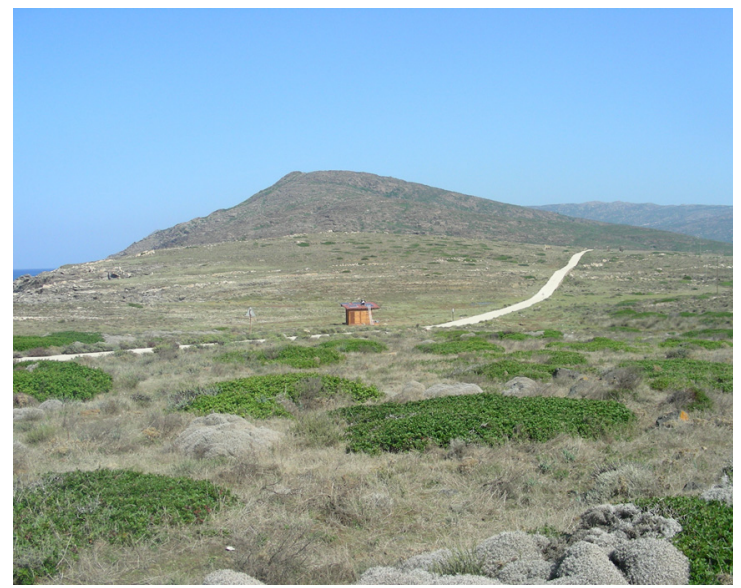

Figure 4: $\quad$ Landscape view of the infopoint in Asinara Island.
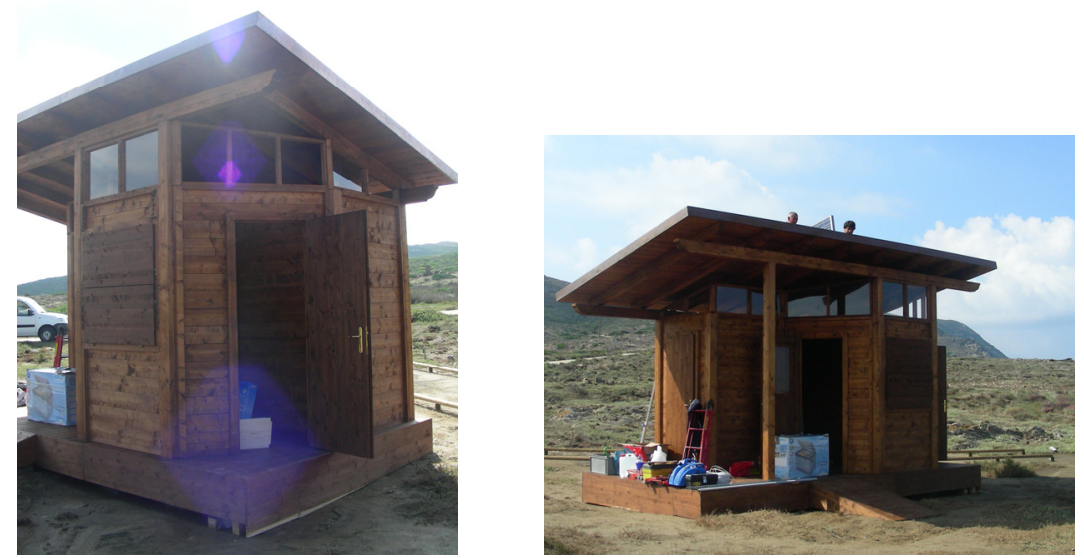

Figure 5: Details of the wood structure and the ventilated roof.

completely energetically self supported, due to the lack of electric and water supply in the area [4].

The final result of the design consists in a light structure realized with a wide use of prefabricated wood elements supporting a photovoltaic plant on the roof able to supply all the technological equipments and the lighting devices.

In detail the $16 \mathrm{mq}$ bungalow (medium height of 3 meters) hosts an infopoint with two PC and an information screen and, in the opposite site, a restoration desk and a toilette for small groups of tourists.

The structure has no foundation - the Sardinia islands is the only Italian region with no seismic requirements - but is able to stand against $120 \mathrm{~km} / \mathrm{h}$ strong winds due to its wide base platform, $30 \mathrm{~cm}$ high, filled with local heavy stones as shown in the picture taken during the construction phase. The roof, 
open to ventilation, is able to guarantee good performances for air recirculation and spring and summer free cooling and the photovoltaic plant of about $1,3 \mathrm{kWp}$ (24V) supplies power for all the lightning equipments, 2 personal computers, a wide screen and 2 refrigerators for food and beverage.

The project has been realized with the financial contribution of the Ente Parco dell'Asinara.

\section{Conclusion}

The final aim of the two pilot projects is the evaluation of the effectiveness of the combination of the installed technologies, following the global approach of the sustainable design, in a Mediterranean climate, measuring the reduction of $\mathrm{CO} 2$ emissions and of land resource consumption during the first year of life of the structures. In order to evaluate a global result a monitoring campaign based on four seasonal measurements is being performed by the Department of Fisica Tecnica together with Italian Environmental Ministry and the Ente Parco of Asinara Island. Final consideration will be available in October 2010.

\section{References}

[1] Ardito S., Parks of Italy - the protected areas system, Italian Environmental Ministry - Carsa Edizioni, 2004, 226-230 - abstract

[2] Cinquepalmi F., Gugliermetti, F, Cumo, F., Technologies for sustainable building the pilot project of the municipality of Rome, Palombi and partner $2008-1$

[3] Berta L., Bovati M., Progettare con legno - Maggioli editore 2007 - 2

[4] De Santoli L., Moncada G., fondamenti di sostenibilità energeticoambientale, 2008 - 3 